\title{
Folate-targeted polymeric micelles loaded with ultrasmall superparamagnetic iron oxide: combined small size and high MRI sensitivity
}

This article was published in the following Dove Press journal:

International Journal of Nanomedicine

9 June 2012

Number of times this article has been viewed

\section{Guo-bin Hong',2 \\ Jing-xing Zhou ${ }^{2}$ \\ Ren-xu Yuan ${ }^{3}$}

'Department of Radiology, Fifth Affiliated Hospital, Zhuhai,

${ }^{2}$ Department of Radiology, Sun Yatsen Memorial Hospital, Guangzhou,

${ }^{3}$ School of Chemistry and Chemical Engineering, Sun Yat-sen University,

Guangzhou, China
Correspondence: Jing-xing Zhou Department of Radiology, Sun Yat-sen Memorial Hospital, Sun Yat-sen University, Guangzhou 510120, China

Tel +862081332243

Fax +86 2081332702

Email gdzhoujx@163.com
Abstract: Targeted delivery of contrast agents is a highly desirable strategy for enhancing diagnostic efficiency and reducing side effects and toxicity. Water-soluble and tumor-targeting superparamagnetic iron oxide nanoparticles (SPIONs) were synthesized by loading hydrophobic SPIONs into micelles assembled from an amphiphilic block copolymer poly(ethylene glycol)poly( $\varepsilon$-caprolactone) (PEG-PCL) bearing folate in the distal ends of PEG chains. Compared to the water-soluble SPIONs obtained by small molecular surfactant coating, ultrasmall SPION encapsulation with PEG-PCL micelles (PEG-PCL-SPIONs) simultaneously increases transverse $\left(r_{2}\right)$ and decreases longitudinal $\left(r_{1}\right)$ magnetic resonance (MR) relaxivities of water proton in micelle solution, leading to a notably high $r_{2} / r_{1}$ ratio up to 78 , which makes the PEG-PCL-SPIONs a highly sensitive MR imaging (MRI) $T_{2}$ contrast agent. The mean size of folate-attached SPION micelles (Fa-PEG-PCL-SPIONs) is $44 \pm 3 \mathrm{~nm}$ on average, ideal for in vivo MRI applications in which long circulation is greatly determined by small particle size and is highly desirable. Prussian blue staining of BEL-7402 cells over-expressing folate receptors, after incubation with micelle-containing medium, demonstrated that folate functionalization of the magnetic particles significantly enhanced their cell uptake. The potential of Fa-PEG-PCL-SPIONs as a potent MRI probe for in vivo tumor detection was assessed. At 3 hours after intravenous injection of the Fa-PEG-PCL-SPION solution into mice bearing subcutaneous xenografts of human BEL-7402 hepatoma, a 41.2\% signal intensity decrease was detected in the $T_{2}$-weighted MR images of the tumor, indicating the efficient accumulation of Fa-PEG-PCL-SPIONs in the tumor tissue.

Keywords: tumor targeting, magnetic resonance imaging, polymeric micelles, superparamagnetic iron oxide

\section{Introduction}

As one of the most powerful diagnostic techniques in clinical medicine, magnetic resonance imaging $(\mathrm{MRI})$ has attracted much attention, and considerable effort has been made to improve its resolution and contrast quality in recent years. ${ }^{1}$ Targeted delivery of contrast agents is a highly desirable strategy for simultaneously enhancing contrast effect and reducing side effects and toxicity. This technique is extremely useful for detecting early signs of diseases. Superparamagnetic nanocrystals are well-known excellent MRI probes which can noninvasively monitor in vivo events even at molecular and cellular level. To date, most interest has been focused on superparamagnetic iron oxide nanoparticles (SPIONs), magnetite $\left(\mathrm{Fe}_{3} \mathrm{O}_{4}\right)$ and maghemite $\left(\gamma-\mathrm{Fe}_{2} \mathrm{O}_{3}\right){ }^{2}$

Magnetite nanoparticles are usually prepared through a conventional coprecipitation method in the aqueous phase. Although this method is suitable for mass 
production of magnetic nanoparticles, ${ }^{3}$ it requires careful adjustment of the $\mathrm{pH}$ value of the solution for particle formation and stabilization, and it is difficult to control size and size distribution, particularly when the desired particle size is smaller than $20 \mathrm{~nm}$. By contrast, high-temperature decomposition strategies have been developed recently to produce monodisperse and highly crystalline iron oxide nanoparticles. ${ }^{3,4}$ Magnetic nanoparticles produced by high-temperature decomposition are potentially more advantageous for biomedical applications such as magnetic resonance imaging and magnetic cell separation, in which well-defined particle size and uniform size distribution are often required to achieve long circulation or efficient cell uptake. However, nanoparticles thus prepared are coated with a layer of surfactant molecules to prevent them from aggregation. The long hydrocarbon chains of the surfactants covering the SPIONs lead to high hydrophobicity of particle surface, hence the biological applications of these nanoparticles are greatly restricted because of their poor dispersibility in aqueous solution. ${ }^{5}$ Furthermore, particles that have a highly hydrophobic surface can be efficiently coated with plasma components and thus rapidly removed from the circulation. Therefore, surface modification of these magnetic iron oxide nanocrystals is essential for most of their bio-related applications. ${ }^{6}$ An ideal surface modification should (a) stabilize the nanoparticles in biological surroundings with a $\mathrm{pH}$ of about 7.4 and particularly at physiological salt concentration, (b) provide functional groups at the particle surface for further derivatization (eg, attaching receptor ligands for cell-specific uptake), and (c) suppress the uptake of nanoparticles by the reticuloendothelial system. ${ }^{7}$ So far, several methods have been explored for converting hydrophobic SPIO nanocrystals into hydrophilic ones, typically including ligand exchange, ${ }^{8,9}$ bipolar molecule, ${ }^{5}$ and polymer stabilization. ${ }^{12}$ These modifications have the advantages of avoiding potential exposure of hydrophobic SPION surface and adsorption of blood proteins, and may allow a prolonged blood circulation. Nowadays, more interest is being focused on amphiphilic polymers because of their advantage of high colloidal stability over small molecular surfactants. For example, hydrophobic iron oxide nanoparticles were encapsulated within an amphiphilic polymer shell consisting of poly-(maleic anhydride-alt-1-tetradecene) and cross-linked by bis(6-aminohexyl)amine, and ABA-type triblock copolymer consisting of poly(propylene oxide) and poly(ethylene oxide) (Pluronic F127) was also used to transfer the hydrophobic SPIONs into water-soluble ones. ${ }^{11}$ In addition, Ai et al reported that clusters of hydrophobic magnetic nanoparticles could be encapsulated into the hydrophobic core of polymeric micelle, resulting in an ultrasensitive MRI $T_{2}$ contrast agent. ${ }^{12}$ These approaches have been demonstrated successfully for preparing magnetic nanoparticles with high monodispersity and stability in aqueous solution. It is noted that water-soluble SPIO nanoparticles reported in such work mostly lack in specificity towards a pathological site, which potentially limits their in vivo applications. Indeed, most of the convincing successes in in vivo applications of SPIO-based nanoparticles as MRI probes have been achieved with nanoparticles smaller than $50 \mathrm{~nm} \cdot{ }^{13,14}$ Further research towards a combination of reducing particle size for long circulation, enhancing MRI sensitivity, and introducing molecular targeting to guide site-specific delivery is much needed.

In this study, we developed a type of tumor-targeted and water-dispersible SPIO nanoparticle smaller than $50 \mathrm{~nm}$ in diameter. By loading hydrophobic SPIO nanocrystals into polymeric micelles assembled from a diblock copolymer of polyethylene glycol (PEG) and biodegradable poly- $\varepsilon$ caprolactone (PCL), we were able to load ultrasmall SPIO nanocrystal into a micelle core, thus decreasing the particle size significantly. Because folic acid receptor is overexpressed in a wide range of cancer cells, ${ }^{15}$ and folate-functionalized PEG-PCL micelles have been used for targeting delivery of a multidrug-resistance modulator, FG020326, ${ }^{16}$ we attached folate onto the surface layer of the polymeric micelles to achieve functional tumor-targeting micelles. We further examined and evaluated the MRI $T_{2}$ sensitivity of the ultrasmall SPIO-loaded targeting micelles (Fa-PEG-PCL-SPION), and tested their potential use as a potent MRI $T_{2}$ probe for in vivo tumor detection on a $1.5 \mathrm{~T}$ clinical MRI scanner.

\section{Materials and methods Materials}

$\varepsilon$-Caprolactone ( $\varepsilon$-CL; Sigma-Aldrich, St Louis, MO) and allyl alcohol (Guangzhou Chemical Reagent Factory, Guangzhou, China) were both purified by vacuum distillation over calcium hydride $\left(\mathrm{CaH}_{2}\right)$. Tetrahydrofuran (THF; Sigma-Aldrich) was dried by refluxing the chemical over a sodium-potassium alloy and distilled under dry argon. 18-Crown-6 (Sigma-Aldrich) was vacuum-dried overnight at $46^{\circ} \mathrm{C}$. 2-Aminoethanethiol hydrochloride, folic acid, $\mathrm{N}$-hydroxysuccinimide (NHS), naphthalene, potassium persulfate $\left(\mathrm{K}_{2} \mathrm{~S}_{2} \mathrm{O}_{8}\right)$, and dicyclohexylcarbodiimide (DCC) were purchased from Sigma-Aldrich. Ethylene oxide (EO, 99\% purity), stored inside a gas tank, was obtained from Foshan Kedi Gas Chemical Industry (Foshan, China). 
Phenyl ether (99\%), benzyl ether (99\%), 1,2-hexadecanediol (97\%), oleic acid (99\%), oleylamine (>70\%) and iron (III) acetylacetonate were purchased from Sigma-Aldrich and used without further purification. BEL-7402 cells, a human hepatic carcinoma cell line with overexpressed surface receptors for folic acid, were obtained from the Experimental Animal Center of Sun Yat-sen University.

\section{Methods}

\section{Synthesis of allyl-terminated diblock copolymer} of PCL and PEG (allyl-PEG-PCL)

The copolymer was synthesized by sequential anionic ringopening polymerization of EO and $\varepsilon$-CL in one pot using potassium alkoxide as an initiator. ${ }^{16}$ THF solution $(4 \mathrm{~mL})$ of potassium naphthalide was allowed to mix with $0.5 \mathrm{~mL}$ allyl alcohol, and then the mixture was stirred for 30 minutes into a flame-dried reaction flask equipped with a magnetic stirring bar and two capillary gas inlets for EO and argon, respectively. Subsequently, $20 \mathrm{~mL}$ anhydrous THF and $1.5 \mathrm{~g}$ 18-crown- 6 predissolved in $5 \mathrm{~mL}$ anhydrous THF in another flamed flask were then transferred into the first reaction flask under argon. After stirring for another 30 minutes, the mixture was cooled with a salted ice-water bath of $-5^{\circ} \mathrm{C}$. A precalculated amount of dry EO was slowly blown and condensed into the reaction mixture. Afterwards, the EO polymerization was conducted at $0^{\circ} \mathrm{C}$ for 24 hours and then at room temperature for 3 days to ensure a thorough conversion of EO. In the second step, a predesigned amount of $\varepsilon-C L$ was injected into the reaction flask under argon protection and then polymerized at room temperature for 48 hours. The polymerization was finally quenched by adding a small amount of acetic acid. The crude copolymer collected by precipitation in hexane was redissolved in dichloromethane and added to tenfold diethyl ether under vigorous stirring. A white powder was sequentially isolated by filtration and washed with hexane and diethyl ether.

${ }^{1} \mathrm{H}-\mathrm{NMR}\left(\mathrm{CDCl}_{3}\right): \delta=1.40\left(\mathrm{~m}, 2 \mathrm{H},-\mathrm{COCH}_{2} \mathrm{CH}_{2} \mathrm{CH}_{2}\right.$ $\left.\mathrm{CH}_{2} \mathrm{CH}_{2} \mathrm{O}-\right), 1.65$ (m, 4H, $-\mathrm{COCH}_{2} \mathrm{CH}_{2} \mathrm{CH}_{2} \mathrm{CH}_{2} \mathrm{CH}_{2} \mathrm{O}-$ ), $2.31\left(\mathrm{t}, 2 \mathrm{H},-\mathrm{COCH}_{2} \mathrm{CH}_{2} \mathrm{CH}_{2} \mathrm{CH}_{2} \mathrm{CH}_{2} \mathrm{O}-\right), 3.65$ (s, $\left.4 \mathrm{H},-\mathrm{CH}_{2} \mathrm{CH}_{2} \mathrm{O}-\right), 4.05\left(\mathrm{t}, 2 \mathrm{H},-\mathrm{COCH}_{2} \mathrm{CH}_{2} \mathrm{CH}_{2} \mathrm{CH}_{2}\right.$ $\left.\mathrm{CH}_{2} \mathrm{O}-\right), 4.2\left(\mathrm{~s}, 2 \mathrm{H},-\mathrm{CH}_{2} \mathrm{CH}_{2} \mathrm{OCO}-\right), 5.15 \sim 5.30(\mathrm{q}, 1 \mathrm{H}$, $\left.\mathrm{CH}_{2}=\mathrm{CH}^{-}\right), 5.80\left(\mathrm{~m}, 2 \mathrm{H}, \mathrm{CH}_{2}=\mathrm{CH}-\right)$.

\section{Conversion of allyl-PEG-PCL into $\mathrm{NH}_{2}$-PEG-PCL}

The reaction was conducted in an aqueous micelle solution, which was prepared by slowly adding a THF solution $(2 \mathrm{~mL})$ of allyl-PEG-PCL $(0.5 \mathrm{~g})$ into distilled water $(20 \mathrm{~mL})$ under stirring, and then allowing evaporation of THF and formation of micelles. The micelle solution was first bubbled with nitrogen for 1 hour to remove oxygen, and then $\mathrm{K}_{2} \mathrm{~S}_{2} \mathrm{O}_{8}(0.8$ molar equivalent of allyl-PEG-PCL) and 2-aminoethanethiol hydrochloride (tenfold molar equivalent of allyl-PEG-PCL) were added to the above solution. Subsequently, the micelle solution was sealed in a nitrogen atmosphere and stirred for 5 hours at $52^{\circ} \mathrm{C}$. Unreacted 2-aminoethanethiol hydrochloride and $\mathrm{K}_{2} \mathrm{~S}_{2} \mathrm{O}_{8}$ were removed by dialysis against water for 24 hours at room temperature (MW cutoff: $8 \mathrm{kDa}$ ). The obtained micelle solution was immediately freeze-dried (yield $\geq 78 \%$ ).

${ }^{1} \mathrm{H}-\mathrm{NMR}\left(\mathrm{CDCl}_{3}\right): \delta=1.40\left(\mathrm{~m}, 2 \mathrm{H},-\mathrm{COCH}_{2} \mathrm{CH}_{2} \mathrm{CH}_{2}\right.$ $\left.\mathrm{CH}_{2} \mathrm{CH}_{2} \mathrm{O}-\right), 1.65$ (m, 4H, $-\mathrm{COCH}_{2} \mathrm{CH}_{2} \mathrm{CH}_{2} \mathrm{CH}_{2} \mathrm{CH}_{2} \mathrm{O}-$ ), $2.31\left(\mathrm{t}, 2 \mathrm{H},-\mathrm{COCH}_{2} \mathrm{CH}_{2} \mathrm{CH}_{2} \mathrm{CH}_{2} \mathrm{CH}_{2} \mathrm{O}-\right), 2.65 \sim 2.70$ (m, $4 \mathrm{H},-\mathrm{CH}_{2} \mathrm{SCH}_{2}-$ ), 2.94 (t, 3H, $\mathrm{H}_{2} \mathrm{NCH}_{2} \mathrm{CH}_{2} \mathrm{~S}-$ ), 3.65 (s, $\left.4 \mathrm{H},-\mathrm{CH}_{2} \mathrm{CH}_{2} \mathrm{O}-\right) 4.05$ (t, $2 \mathrm{H},-\mathrm{COCH}_{2} \mathrm{CH}_{2} \mathrm{CH}_{2} \mathrm{CH}_{2}$ $\left.\mathrm{CH}_{2} \mathrm{O}^{-}\right), 4.2\left(\mathrm{~s}, 2 \mathrm{H},-\mathrm{CH}_{2} \mathrm{CH}_{2} \mathrm{OCO}-\right)$.

\section{Preparation of folate-conjugated copolymer (folate-PEG-PCL)}

Briefly, folic acid (1 g) dissolved in anhydrous dimethyl sulfoxide (DMSO; $30 \mathrm{~mL}$ ) was reacted overnight with NHS $(0.9 \mathrm{~g})$ in the presence of DCC $(0.5 \mathrm{~g})$ under argon at room temperature, and 1,3-dicyclohexylurea (DCU) was removed by filtration. Subsequently, the above activated folate solution $(3 \mathrm{~mL}$ ) was added to a DMSO solution $(5 \mathrm{~mL})$ containing $\mathrm{NH}_{2}$-PEG-PCL $(0.4 \mathrm{~g})$ and triethylamine $(0.05 \mathrm{~mL})$. The reaction was performed at room temperature for 10 hours under argon. The resulting solution was centrifuged and filtered. The filtrate thus obtained was dialyzed against water for 24 hours (MW cutoff: $1 \mathrm{kDa}$ ). The aqueous solution inside the dialysis bag was then freeze-dried. The powdery sample was redissolved in THF ( $3 \mathrm{~mL}$ ), and the filtrate was added dropwise to distilled water under stirring. After overnight evaporation of THF, the resultant micelle solution was dialyzed against water for 5 days to completely remove unreacted folic acid and any residual THF. The micelle solution was finally freeze-dried to yield a solid powder (yield $\geq 82 \%$ ). To evaluate the conversion rate of $\mathrm{NH}_{2}$-PEG-PCL into folatePEG-PCL, copolymer was dissolved in DMSO and folate absorbance at $363 \mathrm{~nm}$ was measured by a Unico (Shanghai, China) UV-2000 UV-Vis spectrophotometer to quantify the folate mass content in the sample. Absorbance of folate at $363 \mathrm{~nm}$ in DMSO with various concentrations was measured to generate a calibration curve.

${ }^{1} \mathrm{H}-\mathrm{NMR}\left(\mathrm{DMSO}-\mathrm{d}_{6}\right): \delta=1.40\left(\mathrm{~m}, 2 \mathrm{H},-\mathrm{COCH}_{2} \mathrm{CH}_{2} \mathrm{CH}_{2}\right.$ $\left.\mathrm{CH}_{2} \mathrm{CH}_{2} \mathrm{O}-\right), 1.65\left(\mathrm{~m}, 4 \mathrm{H},-\mathrm{COCH}_{2} \mathrm{CH}_{2} \mathrm{CH}_{2} \mathrm{CH}_{2} \mathrm{CH}_{2} \mathrm{O}-\right.$ ), $2.31\left(\mathrm{t}, 2 \mathrm{H},-\mathrm{COCH}_{2} \mathrm{CH}_{2} \mathrm{CH}_{2} \mathrm{CH}_{2} \mathrm{CH}_{2} \mathrm{O}-\right), 2.65 \sim 2.70$ 
(m, 4H, $\left.-\mathrm{CH}_{2} \mathrm{SCH}_{2}-\right), 3.2\left(\mathrm{t}, 3 \mathrm{H}, \mathrm{H}_{2} \mathrm{NCH}_{2} \mathrm{CH}_{2} \mathrm{~S}-\right), 3.65$ (s, 4H, $\left.-\mathrm{CH}_{2} \mathrm{CH}_{2} \mathrm{O}-\right), 4.05\left(\mathrm{t}, 2 \mathrm{H},-\mathrm{COCH}_{2} \mathrm{CH}_{2} \mathrm{CH}_{2} \mathrm{CH}_{2-}\right.$ $\mathrm{CH}_{2} \mathrm{O}-$ ), 4.2 (s, 2H, $\left.-\mathrm{CH}_{2} \mathrm{CH}_{2} \mathrm{OCO}-\right)$, 4.45 (d, 2H, $\mathrm{C}_{9}-\mathrm{H}_{2}$ of folic acid), 6.61 (d, 2H, aromatic protons of folic acid), $7.60(\mathrm{~d}, 2 \mathrm{H}$, aromatic protons of folic acid), $8.62(\mathrm{~s}, 1 \mathrm{H}$, $\mathrm{C}_{7}-\mathrm{H}$ of folic acid).

\section{Synthesis of hydrophobic $\mathrm{Fe}_{3} \mathrm{O}_{4}$ nanoparticles} (as synthesized SPIONs)

$\mathrm{Fe}_{3} \mathrm{O}_{4}$ nanoparticles (SPIO) were synthesized according to a previously reported method. ${ }^{3}$ Briefly, iron (III) acetylacetonate (2 mmol), 1,2-hexadecanediol (10 mmol), oleic acid (6 mmol), oleylamine (6 mmol), and benzyl ether $(20 \mathrm{~mL})$ were mixed and magnetically stirred under a flow of nitrogen. The mixture was heated to $200^{\circ} \mathrm{C}$ for 2 hours and then, under a blanket of nitrogen, heated to reflux $\left(300^{\circ} \mathrm{C}\right)$ for 1 hour. The black mixture was cooled to room temperature by removing the heat source. The product, 6-nm $\mathrm{Fe}_{3} \mathrm{O}_{4}$ nanoparticles, was then precipitated with ethanol, centrifuged (6000 rpm, 10 minutes) to remove the solvent, and redispersed into hexane. A black-brown hexane dispersion of 6-nm $\mathrm{Fe}_{3} \mathrm{O}_{4}$ nanoparticles was then produced.

\section{Synthesis of hydrophilic $\mathrm{Fe}_{3} \mathrm{O}_{4}$ nanoparticles}

(WSPIOs) by small molecular surfactant coating

These hydrophilic $\mathrm{Fe}_{3} \mathrm{O}_{4}$ nanoparticles were synthesized according to a previous report. ${ }^{3}$ Under ambient conditions, a hexane dispersion of hydrophobic $\mathrm{Fe}_{3} \mathrm{O}_{4}$ nanoparticles (about $20 \mathrm{mg}$ in $0.2 \mathrm{~mL}$ ) was added to a suspension of tetramethylammonium 11-aminoundecanoate (about $20 \mathrm{mg}$ in $2 \mathrm{~mL}$ ) in dichloromethane. The mixture was shaken for about 20 minutes, during which time the particles precipitated and were separated using a magnet. The solvent and nonmagnetic suspensions were decanted, and the precipitate was washed once with dichloromethane and separated again using a magnet to remove excess surfactants before drying under $\mathrm{N}_{2}$. The product was then dispersed in deionized water.

\section{Preparation of PEG-PCL coated $\mathrm{Fe}_{3} \mathrm{O}_{4}$ nanoparticles} PEG-PCL coated $\mathrm{Fe}_{3} \mathrm{O}_{4}$ nanoparticles (PEG-PCL-SPIONs) were prepared by solvent evaporation method. Briefly, $20 \mathrm{mg}$ of allyl-PEG-PCL or Fa-PEG-PCL (a mixture of allyl-PEGPCL and folate-PEG-PCL containing 20\% folate-PEG-PCL) and $2 \mathrm{mg}$ SPIO were dissolved in THF (1.0 mL). The above solution was slowly added into $10 \mathrm{~mL}$ of deionized water under sonication and stirred for 48 hours at room temperature to remove THF. These particles were separated by magnetic field-guided accumulation and washed with water to remove excessive polymer. Nanoparticles were resuspended in water by sonication and centrifuged at $3000 \mathrm{rpm}$ for 10 minutes at room temperature to remove any large aggregates. The PEGPCL-SPIONs formulations are shown in Table 1.

\section{Dynamic light scattering (DLS) measurements}

The diameter of PEG-PCL-SPIONs was examined at $25^{\circ} \mathrm{C}$ using a Brookhaven (Holtsville, NY) laser light-scattering system consisting of a BI-200SM goniometer and a BI-9000AT digital correlator. A 532-nm vertically polarized argon ion laser was used as the light source.

\section{TEM measurements}

Transmission electron microscopy (TEM) images were obtained at room temperature in a JEOL (Tokyo, Japan) JEM-2010HR operated at $160 \mathrm{kV}$. Samples for TEM analysis were prepared by depositing a drop of the solution onto carbon-coated copper grids and dried at room temperature for 24 hours. To avoid highly concentrated regions, we scanned the samples near the border of the drop.

\section{Determination of SPIO-loading contents}

The SPIO loading density of PEG-PCL-SPIONs was determined using a polarized Zeeman Atomic Absorption Spectrophotometer (Z-2000; Hitachi High Technologies, Tokyo, Japan). Briefly, PEG-PCL-SPIONs were first weighed before being suspended in $1 \mathrm{M} \mathrm{HCl}$ solution to allow for polymer degradation and complete dissolution of PEG-PCL-SPIONs. Iron concentration was determined at the specific Fe-absorption wavelength $(248.3 \mathrm{~nm})$ based on a previously established calibration curve. SPIO loading density was calculated as the ratio of iron oxide over the total weight of PEG-PCL-SPIONs.

\section{Magnetization measurements}

The magnetization data of PEG-PCL-SPIONs were determined using a MPMS XL-7 Quantum Design (San Diego, CA)

Table I Properties of SPIONs with different formulations

\begin{tabular}{llllll}
\hline Micelle formulation & $\begin{array}{l}\text { Micelle size } \\
(\mathbf{n m})\end{array}$ & $\begin{array}{l}\mathbf{F e}_{3} \mathbf{O}_{4} \text { content } \\
(\mathbf{w t} \%)\end{array}$ & $\boldsymbol{r}_{1}$ & $\boldsymbol{r}_{2}$ & $\boldsymbol{r}_{2} / \boldsymbol{r}_{1}$ \\
\hline Fa-PEG-PCL-SPIONs $^{\mathrm{a}}$ & $44 \pm 3$ & $33.5 \%$ & 1.7 & $\mathrm{II} 3$ & 66 \\
PEG-PCL-SPIONs & $38 \pm 3$ & $37.0 \%$ & 1.4 & 110 & 78 \\
Hydrophobic SPIO & 6 & $77.0 \%$ & $\mathrm{nd}$ & $\mathrm{nd}$ & $\mathrm{nd}$ \\
WSPIOs $^{\mathrm{c}}$ & 6 & & 3.2 & 40 & 12.5 \\
Resovit $^{\mathrm{b}}$ & 65 & & 7.2 & 82 & 11.3 \\
\hline
\end{tabular}

Notes: $r_{1}$ and $r_{2}$ values are expressed as Fe $\mathrm{mM}^{-1} \mathrm{~s}^{-1}$. aSurface layer was a mixture of Fa-PEG-PCL and PEG-PCL containing 20\% Fa-PEG-PCL; ${ }^{\mathrm{b} T e s t e d ~ b y ~ T G A ; ~ ' H y d r o p h i l i c ~}$ SPIO obtained by small molecular surfactant coating.

Abbreviations: Fa-PEG-PCL-SPIONs, folate-attached poly(ethylene glycol)-poly( $\varepsilon$ caprolactone) superparamagnetic iron oxide nanoparticles; WSPIOs, water-soluble superparamagnetic iron oxide nanoparticles; nd, not determined. 
SQUID magnetometer at $10 \mathrm{~K}$ and $300 \mathrm{~K}$. The applied magnetic field was varied from $2 \times 10^{4}$ Oe to $-2 \times 10^{4}$ Oe in order to generate hysteresis loops.

\section{In vivo $M R$ imaging}

Animals (nu/nu CD-1 male nude mice each weighing $25 \pm 3 \mathrm{~g}$ ) were maintained on a folate-free diet for 1 week before MR imaging. Subcutaneous tumor xenografts were generated in anesthetized (10\% chloralhydrate) mice by subcutaneous injection of $1 \times 10^{7}$ BEL-7402 cells in $200 \mu \mathrm{L}$ of serum-free cell culture medium. Animals were studied by MR imaging when the subcutaneous xenografts reached a diameter of about $1 \mathrm{~cm}$. Nanoparticle aqueous solution in $0.9 \%$ soldium chloride was injected into a nude mouse bearing the BEL-7402 tumor through the tail vein. The applied dose was $10 \mathrm{mg} \mathrm{Fe} / \mathrm{kg}$ of body weight, which is similar to that recommended for commercial contrast agents such as Resovist. The animals were scanned under an Intera $1.5 \mathrm{~T}$ MR scanner (Philips, Amsterdam, Netherlands) at room temperature. A home-built $4.5 \times 9$-cm linearly polarized birdcage radio frequency coil was used for all studies. The $T_{2}$-weighted echo images were acquired before and after nanoparticle administration using the following parameters: repetition time $\left(T_{\mathrm{R}}\right) /$ echo time $\left(T_{\mathrm{E}}\right), 5000 / 100 \mathrm{~ms}$; field of view, $150 \mathrm{~mm}$; matrix, $256 \times 256$; slice thickness, $1.5 \mathrm{~mm}$. At each experimental time point, six mice were scanned for both targeting and nontargeting groups. The MRI $T_{2}$ signal intensities within the region of interest were measured. After MR imaging, two mice of each group were killed and the tumors were further processed for Prussian blue staining.

\section{Prussian blue staining}

Tumors were fixed in 10\% formalin and cryoprotected with $18 \%$ sucrose solution. Tissues were then snap frozen in OCT medium and sectioned at $8 \mu \mathrm{m}$. Sections were rinsed in distilled water and then incubated for 30 minutes in a solution of $2 \%$ potassium ferrocyanide and $2 \%$ hydrochloric acid in a 1:1 ratio. After Prussian blue staining, the sections were rinsed again and counterstained with $1 \%$ neutral red.

\section{Statistical analysis}

MRI signal intensity and rate of signal intensity variety were expressed as mean \pm standard deviation and median, respectively. One-way analysis of variance was performed to determine significant changes in MRI signal intensity, and the Kruskal-Wallis test was used to determine significant changes in rate of signal intensity variety. If a one-way analysis of variance or Kruskal-Wallis test showed that there was a significant difference among the MRI signal intensity or the rate of signal intensity variety at four time points ( 0 h, 3 h, 6 h, 24 h), post hoc multiple comparison (Student Newman-Keuls test) would be used to determine which time point differed significantly. $P$-values less than 0.05 were considered to be statistically significant. All statistical tests were two-sided and performed using the Statistical Package for the Social Sciences (IBM, Armonk, NY) version 13.0 software for Windows.

\section{Results and discussion Preparation and characterization of folate-targeted and SPIO-loaded micelles} Amphiphilic block copolymers, folate-PEG-PCL $\left(M_{\mathrm{n}}=5.1 \mathrm{kD}\right.$, $\left.M_{\mathrm{n}(\mathrm{PEG})}=2.9 \mathrm{kD}, M_{\mathrm{n}(\mathrm{PCL})}=0.87 \mathrm{kD}\right)$ and allyl-PEG-PCL $\left(M_{\mathrm{n}}=3.8 \mathrm{kD}, M_{\mathrm{n}(\mathrm{PEG})}=2.9 \mathrm{kD}, M_{\mathrm{n}(\mathrm{PCL})}=0.87 \mathrm{kD}\right)$, were used for micelle fabrication. They were synthesized by multistep chemical reactions, as reported in a recent publication, ${ }^{16}$ (Figure 1). Magnetic nanocrystals for MR signal enhancement must be well defined in structure and size because the size, crystalline phase, and stoichiometry of these nanocrystals can affect the MR signals. In this research, $\mathrm{Fe}_{3} \mathrm{O}_{4}$

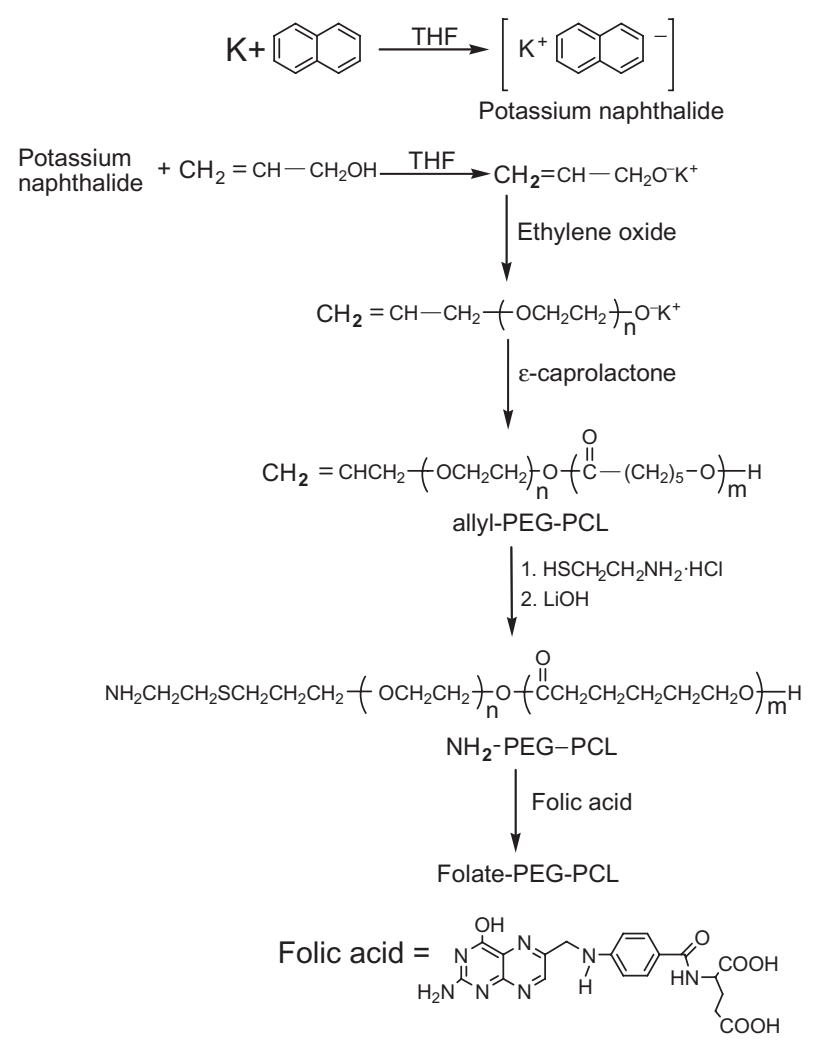

Figure I Synthetic approach of folate-PEG-PCL for SPION coating. Abbreviations: PEG-PCL, poly(ethylene glycol)-poly( $\varepsilon$-caprolactone); SPION, superparamagnetic iron oxide nanoparticle. 
nanoparticles (SPIO) measuring $6 \mathrm{~nm}$ were prepared by the high-temperature decomposition method. ${ }^{3}$ As shown in Figure 3A the SPIO nanoparticles are uniform in shape and size. A comparison between the lattice spacing based on the selected area electron diffraction pattern (Figure 3C) with that for bulk $\mathrm{Fe}_{3} \mathrm{O}_{4}$ indicates that the particle composition is $\mathrm{Fe}_{3} \mathrm{O}_{4}$.

The $\mathrm{Fe}_{3} \mathrm{O}_{4}$ nanoparticles prepared by the high-temperature decomposition method are hydrophobic. A simple but effective method was adopted to make them water soluble and biocompatible. We hypothesized that the hydrophobic segments of PEG-PCL could anchor at the interface of the hydrophobic surfactant shell around iron oxide nanoparticles and the hydrophilic PEG segments extend into the aqueous phase. Figure 2 depicts an amphiphilic copolymer of PEG-PCL being introduced to encapsulate the ultrasmall SPIO nanocrystal, which forms stable micellar dispersion in aqueous media. Figure 3 shows the TEM images of PEG-PCL-coated and-uncoated $\mathrm{Fe}_{3} \mathrm{O}_{4}$ nanocrystals. The PEG-PCL-coated $\mathrm{Fe}_{3} \mathrm{O}_{4}$ nanoparticles retain their original size of $6 \mathrm{~nm}$ without evidence of aggregation (Figure 3B). The diameters of blank PEG-PCL micelle and PEG-PCLSPIONs were determined by dynamic light scattering, and results are shown in Figure 4 and Table 1. The diameter of blank PEG-PCL micelles is about $28 \mathrm{~nm}$, which is smaller than that of PEG-PCL-SPIONs (38 nm). The size of PEGPCL-SPIONs is highly uniform in water and larger than that of SPIO nanoparticles $(6 \mathrm{~nm})$ unencapsulated with polymeric micelle. Since dynamic light-scattering measurement provides information on the hydrodynamic size of particles, including the magnetite core and polymer coating layers, the increase of the particle diameter upon micellar encapsulation apparently can be attributed to the hydrodynamic radius of the polymeric coating on the iron oxide nanoparticles. This result is in agreement with that of Pluronic-coated iron oxide nanoparticles. ${ }^{18}$

The hydrodynamic size of nanoparticles in physiological fluids is known to significantly affect their plasma half-life time, biodistribution, and pharmacokinetic properties. Noting that the capillary diameter of the reticuloendothelial system is about $50 \mathrm{~nm},{ }^{19}$ the in vivo application of nanoparticles would preferentially require sizes smaller than $50 \mathrm{~nm}$ to achieve long circulation. As to the in vivo MRI application, SPIONs matching this size requirement are very useful for imaging of the vascular compartment (magnetic resonance angiography), and for perfusion imaging, neurofunctional imaging, imaging of lymph nodes, receptor imaging, and target-specific imaging. ${ }^{20}$ Although a lot of SPION-related MRI contrast agents have been reported thus far, development of SPIONs combining small size (for example, smaller than $50 \mathrm{~nm}$ ) and high MRI sensitivities is still a hot topic in this field. Based on the previous work that multiple SPIONs loading into polymeric micelle cores resulted in high MRI $T_{2}$ relaxivity and also significant size increase, ${ }^{12}$ the current work successfully reduced particle size by loading ultrasmall SPIONs into polymeric micelle core.

\section{Magnetization and MRI sensitivity of folate-targeted and SPIO-loaded micelles}

The magnetization loops of the $\mathrm{Fe}_{3} \mathrm{O}_{4}$ nanoparticles and PEGPCL-SPIONs measured at both $10 \mathrm{~K}$ and room temperature are shown in Figure 5. Both the $\mathrm{Fe}_{3} \mathrm{O}_{4}$ nanoparticles and PEG-PCL-SPIONs are ferromagnetic at $10 \mathrm{~K}$ with a coercivity of $240 \mathrm{Oe}$ and $146 \mathrm{Oe}$, respectively. At room temperature,

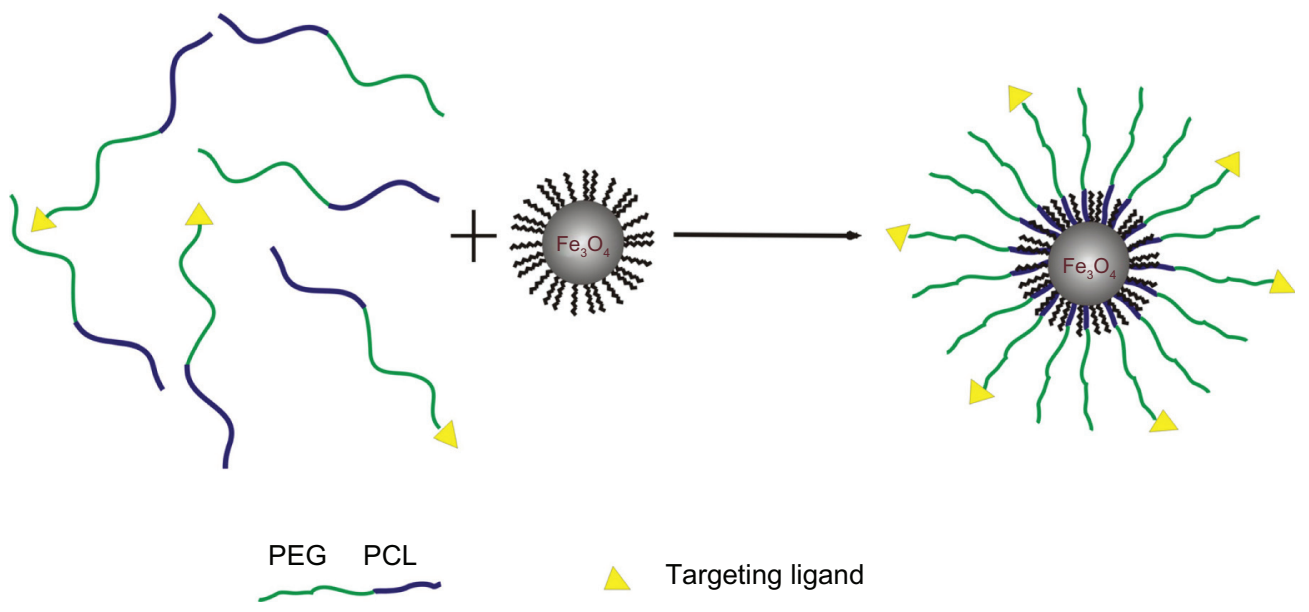

Figure 2 Synthesis of monodisperse $\mathrm{Fe}_{3} \mathrm{O}_{4}$ nanoparticles coated with biodegradable diblock copolymer. Abbreviation: PEG-PCL, poly(ethylene glycol)-poly( $\varepsilon$-caprolactone). 

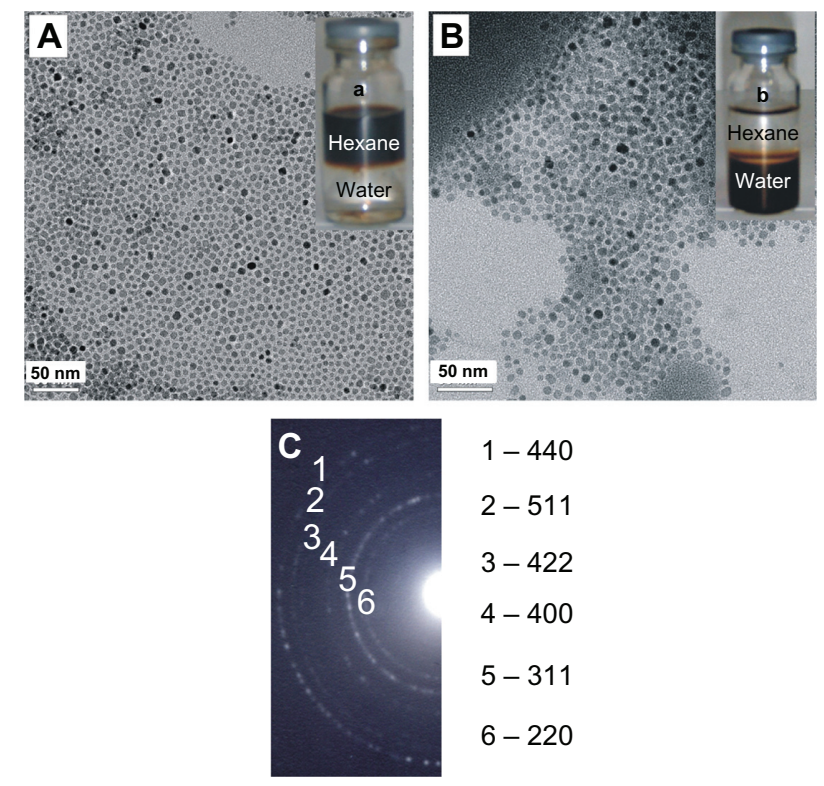

$1-440$
$2-511$
$3-422$
$4-400$
$5-311$
$6-220$

Figure 3 Transmission electron microscopy (TEM) images of synthesized $6 \mathrm{~nm}$ SPIONs (A) and PEG-PCL-SPIONs (B). The insert (a) shows the synthesized SPIONs were soluble in hexane, and PEG-PCL-SPIONs were easily dispersed in water (b). The selected area electron diffraction (SAED) pattern of 6-nm $\mathrm{Fe}_{3} \mathrm{O}$ nanoparticles is shown in (C).

Abbreviation: PEG-PCL-SPIONs, poly(ethylene glycol)-poly( $\varepsilon$-caprolactone) superparamagnetic iron oxide nanoparticles.

the samples show typical superparamagnetic behavior, with zero coercivity and remanence. Under a large external field, the magnetization of the particles aligns with the field direction and reaches its saturation value (saturation magnetization, $\sigma_{\mathrm{s}}$ ). A saturation magnetization of $64.7 \mathrm{Fe}$ emu/g was determined for the $\mathrm{Fe}_{3} \mathrm{O}_{4}$ nanoparticles. The value was $61.3 \mathrm{Fe}$ emu/g for PEG-PCL-SPIONs, indicating no obvious loss in magnetization, which is different from several reports that entrapment of magnetic nanoparticles into hydrophobic polymers such

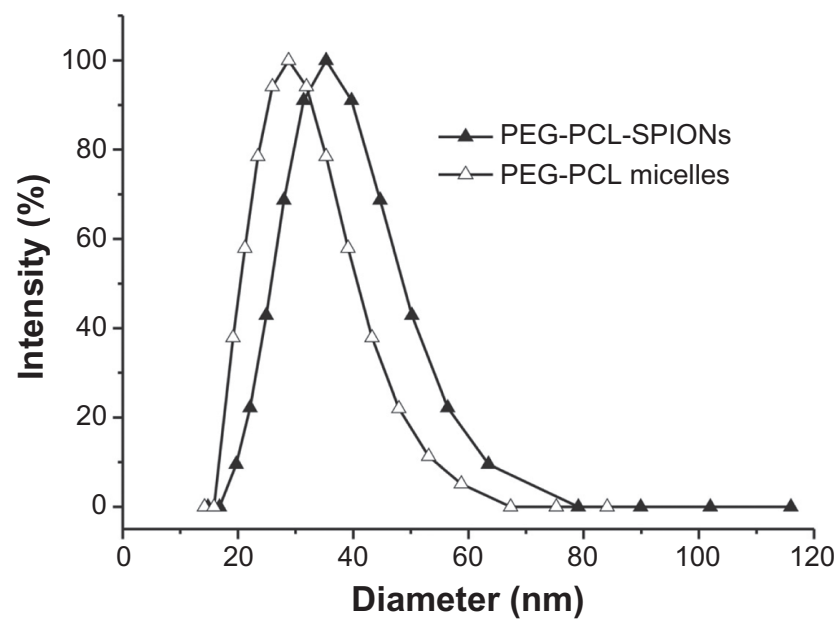

Figure 4 DLS profiles of PEG-PCL-SPIONs and blank PEG-PCL micelle. Abbreviations: DLS, dynamic light scattering; PEG-PCL-SPIONs, poly(ethylene glycol)-poly( $\varepsilon$-caprolactone) superparamagnetic iron oxide nanoparticles.

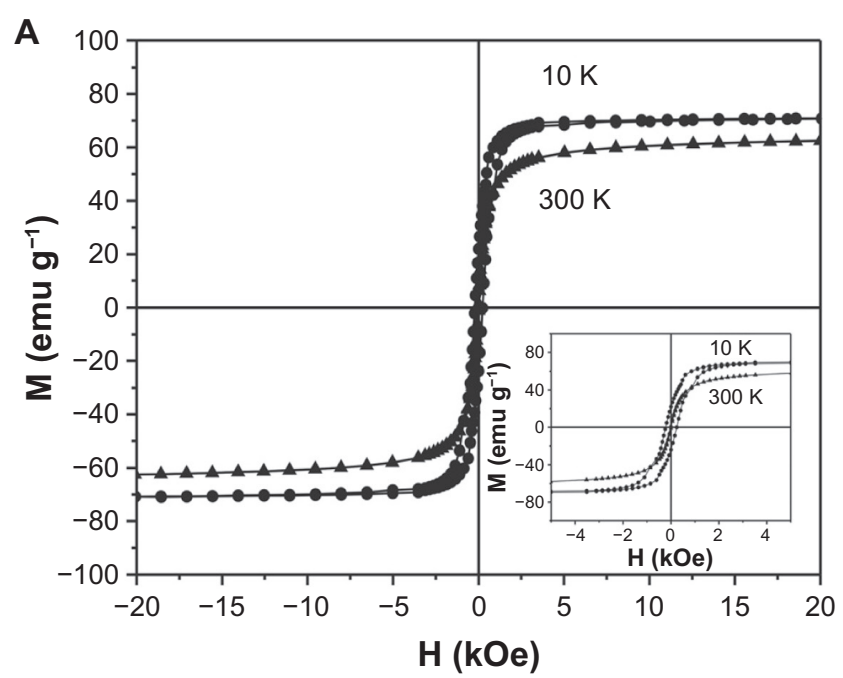

B

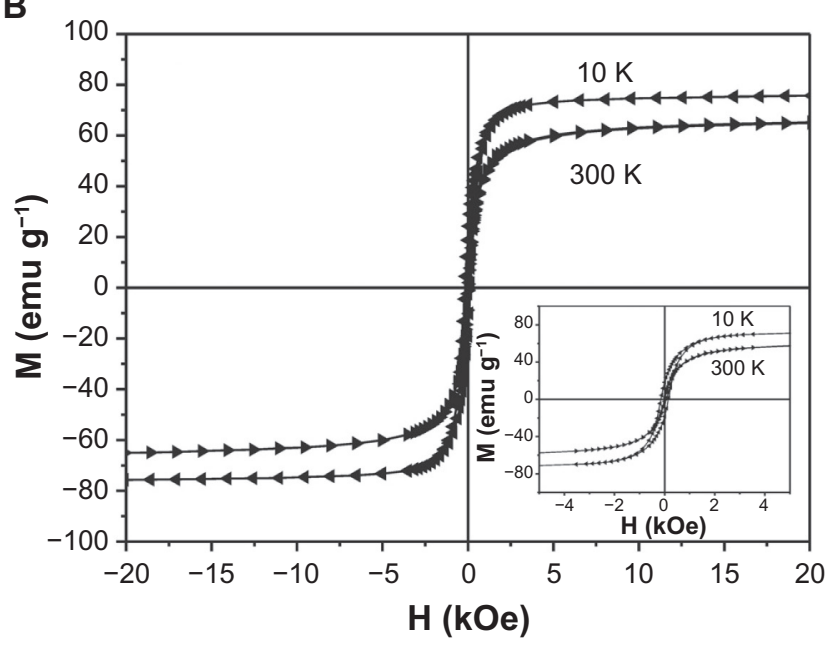

Figure 5 Hysteresis loops of 6-nm $\mathrm{Fe}_{3} \mathrm{O}_{4}$ nanoparticles (A) and PEG-PCL-SPIONs (B) measured at $300 \mathrm{~K}$ and $10 \mathrm{~K}$.

Abbreviation: PEG-PCL-SPIONs, poly(ethylene glycol)-poly(e-caprolactone) superparamagnetic iron oxide nanoparticles.

as poly-DL-lactide-coglycolide and polylactides leads to a loss of saturation magnetization value. ${ }^{21,22}$ The superparamagnetic character of the PEG-PCL coated $\mathrm{Fe}_{3} \mathrm{O}_{4}$ nanoparticles is important for biomedical applications where remanent magnetization is undesirable. As MRI contrast agents, the particles must rapidly relax their magnetic moment vectors to random directions when the applied magnetic field is removed. ${ }^{17}$

In addition to the particle size, MRI sensitivity is another key property determining the application of SPIONs as efficient contrast agents. Based on the MRI mechanism, the image contrast comes from local differences in spin relaxation kinetics of hydrogen nuclei of water along the longitudinal and transverse planes of the main magnetic field applied to the specimen. A contrast agent can alter the signal 
intensity by selectively shortening hydrogen longitudinal (spin-lattice) relaxation time $T_{1}$ and transverse (spin-spin) relaxation time $T_{2}$. The effect of an MRI contrast agent is assessed based on its longitudinal and transverse relaxivities $r_{1}$ and $r_{2}$, which reflect the ability of the contrast agent to alter $T_{1}$ and $T_{2}$, respectively. A $T_{2}$ agent provides enhancement to negative MRI contrast and thus causes darkening of the interfered regions. The higher the $r_{2} / r_{1}$ ratio, the better the effectiveness of a $T_{2}$ agent. Following the size and magnetization characterizations, we measured longitudinal and transverse relaxation times of the water protons $\left(T_{1}\right.$ and $\left.T_{2}\right)$ for PEG-PCL-SPION aqueous solutions. For comparison, water-soluble $\mathrm{Fe}_{3} \mathrm{O}_{4}$ nanoparticles (WSPIOs) prepared by mixing hydrophobic $\mathrm{Fe}_{3} \mathrm{O}_{4}$ nanoparticles with bipolar molecules (tetramethylammonium 11-aminoundecanoate) were also measured. As shown in Table 1, SPION coating with polymeric micelle concomitantly increased $r_{2}$ relaxivities $\left(110 \mathrm{Fe} \mathrm{mM}^{-1} \mathrm{~s}^{-1}\right.$ vs $40 \mathrm{Fe} \mathrm{mM}^{-1} \mathrm{~s}^{-1}$ ) and decreased $r_{1}\left(1.4 \mathrm{Fe} \mathrm{mM}^{-1} \mathrm{~s}^{-1}\right.$ vs $\left.3.2 \mathrm{Fe} \mathrm{mM}^{-1} \mathrm{~s}^{-1}\right)$, leading to significantly elevated $r_{2} / r_{1}$ values (78 vs 13 ). In comparison with the small molecular surfactant coating, SPION coating with polymeric micelle leads to a remarkable $r_{2} / r_{1}$ increase by a factor of six, strongly indicating greatly enhanced MRI $T_{2}$ sensitivity.

It is well known that the process of $T_{1}$ shortening requires direct interaction between protons and the magnetic parts of the contrast agent, and proton $T_{1}$ relaxation in the regions of interest is expedited by easy access of the water molecules to the contrast agent. The $r_{1}$ values of both PEG-PCL-SPION and WSPIO are significantly smaller than 20-30 $\mathrm{Fe} \mathrm{mM}^{-1} \mathrm{~s}^{-1}$, typical for hydrophilic SPION in a dextran matrix (eg, Clariscan, monocrystalline iron oxide nanoparticles [MION]-46). ${ }^{23}$ Unlike MION-46 and other $T_{2}$ contrast agents whose coating layer is entirely hydrophilic, PEG-PCL-SPIONs and WSPIOs in the present research comprise two hydrophobic interlayers, ie, coatings of oleic acid and hydrophobic moiety of small molecular surfactant or polymer, which can impede the water molecules from contacting with the magnetic core and consequently leads to the lower $r_{1}$ value. Furthermore, the formation of a thick coating layer of hydrophobic PCL on the original $\mathrm{Fe}_{3} \mathrm{O}_{4}$ nanoparticles is hypothesized to be the underlying cause for an even lower $r_{1}$ of PEG-PCL-SPIONs compared to WSPIOs, whose hydrophobic coating layer is apparently thinner. $T_{2}$ shortening in the presence of an MRI contrast agent is closely related to the local Fe concentration, particle size, particle surface charge, particle surface functional groups, and the surface area that is accessible to the bulk water. Recent reports have demonstrated that $r_{2}$ values can be increased by clustering $r_{2}$ agents within nanocontainers such as polymeric micelle ${ }^{24}$ and liposome. ${ }^{10}$ In this case, the local Fe concentration is high and maintainable, whatever the dilution factor applied to the solution. This is apparently not a reason leading to $r_{2}$ increase upon coating ultrasmall SPIONs with polymer micelle, because such micellar encapsulation does not change the local Fe concentration, ie, the stable dispersion of nonclustered 6-nm SPIONs in water, as shown in the TEM image Figure 3B. The phenomenon may be explained from the large magnetic field heterogeneity around the nanoparticle through which water molecules diffuse. Diffusion induces dephasing of the proton magnetic moments and results in $T_{2}$ shortening. There exist three types of water molecules in colloidal systems: "bound" water, strongly associated with the polymeric chains by means of hydrogen bonds or polar interactions; "interfacial" water, characterised by hydrophobic interaction with the macromolecule; and "bulk" water, whose properties are not affected by the presence of the polymeric matrix. ${ }^{25} T_{2}$ relaxivity of bound water is very high compared to that of bulk water. For the PEG-PCL-SPION aqueous dispersions, the mobility of water molecules in the diffusing layer is restricted because of hydrogen bonding between the water molecules and the hydrophilic chains of the PEG blocks, resulting in a large amount of bound water, which should be one of the reasons for the high $T_{2}$ relaxivities of PEG-PCL-SPIONs. Note that the $r_{1}$ and $r_{2}$ values of the commercially available $T_{2}$ agent Resovit were 7.2 and $82 \mathrm{Fe}$
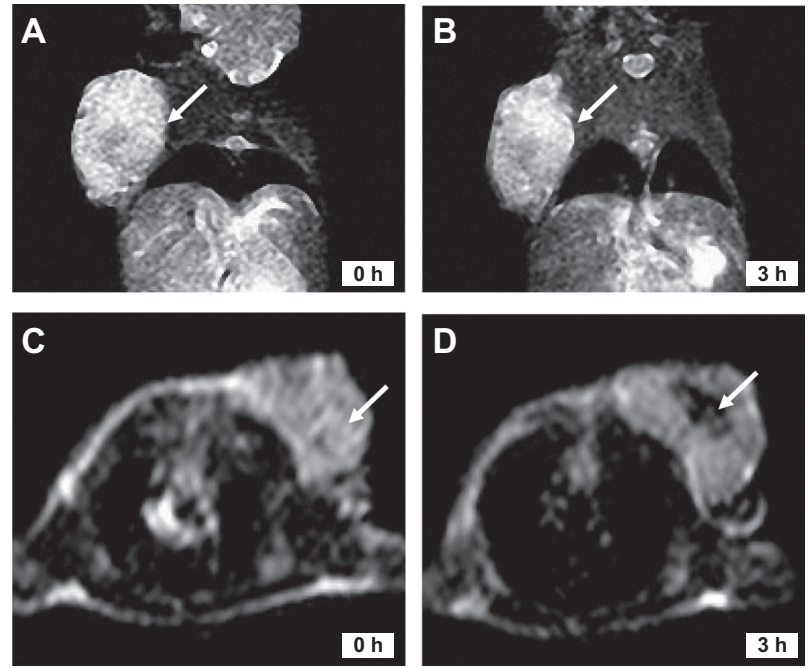

Figure $6 T_{2}$-weighted MRI images $\left(T_{R} / T_{E}, 5000 / 100 \mathrm{~ms}\right)$ taken at 0 and 3 hours after injecting $5 \mathrm{mg}$ of Fe/kg of PEG-PCL-SPIONs (A and B) and Fa-PEG-PCL-SPIONs (C and D) via tail vein into a nude mouse bearing BEL-7402 tumor (about I cm in diameter).

Note: The white arrow indicates the xenograft tumor for determining $T_{2}$-weighted signal intensity change.

Abbreviations: MRI, magnetic resonance imaging; $T_{\mathrm{R}}$, repetition time; $T_{\mathrm{E}}$, echo time; Fa-PEG-PCL-SPIONs, folate-attached poly(ethylene glycol)-poly( $\varepsilon$-caprolactone) superparamagnetic iron oxide nanoparticles. 
Table 2 Dependence of MRI $T_{2}$-weighted signal intensity and signal intensity changes of tumor on the postinjection time

\begin{tabular}{|c|c|c|c|c|}
\hline \multirow[t]{2}{*}{ Hour(s) } & \multicolumn{2}{|l|}{ Fa-PEG-PCL-SPIO } & \multicolumn{2}{|l|}{ PEG-PCL-SPIO } \\
\hline & $\begin{array}{l}\text { MRI signal intensity } \\
(\bar{x} \pm s)\end{array}$ & $\begin{array}{l}\text { MRI signal intensity } \\
\text { changes, } \Delta \text { SI } \\
(\%, M)\end{array}$ & $\begin{array}{l}\text { MRI signal intensity } \\
(\bar{x} \pm s)\end{array}$ & $\begin{array}{l}\text { MRI signal intensity } \\
\text { changes, } \Delta \text { SI } \\
(\%, M)\end{array}$ \\
\hline 0 & $1691 \pm 35$ & 0 & $1822 \pm 84$ & 0 \\
\hline 3 & $998 \pm 30 *$ & $-41.2^{*}$ & $|54| \pm\left. 8\right|^{*}$ & $-16.4^{*}$ \\
\hline 6 & $1145 \pm 71 *$ & $-32.4^{*}$ & $1576 \pm 108^{*}$ & $-12.9^{*}$ \\
\hline 24 & $|503 \pm 8| *$ & $-11.5^{*}$ & $1700 \pm 77$ & -6.43 \\
\hline Statistical value & $F=177.52$ & $\chi^{2}=21.69$ & $F=12.56$ & $x^{2}=19.98$ \\
\hline
\end{tabular}

Notes: $* P<0.05$. At each experimental time point, six mice were scanned for both targeting and nontargeting groups. $\Delta S \mathrm{SI}$ was calculated according to the following equation: $\Delta \mathrm{SI}=\left(\mathrm{SI}_{\text {post }}-\mathrm{SI}_{\text {pre }}\right) / \mathrm{SI}_{\text {pre }} \times 100 \%$, where $\mathrm{SI}_{\text {pre }}$ and $\mathrm{SI}_{\text {post }}$ are signal intensities of pre- and postinjection, respectively.

Abbreviation: Fa-PEG-PCL-SPIO, folate-attached poly(ethylene glycol)-poly( $\varepsilon$-caprolactone) superparamagnetic iron oxide.

$\mathrm{mM}^{-1} \mathrm{~s}^{-1}$, respectively, corresponding to an $r_{2} / r_{1}$ value of 11.3 at $1.5 \mathrm{~T}$ in the manufacturer's product summary. Therefore the high $r_{2} / r_{1}$ value (herein 78) makes PEG-PCL-SPIONs a very promising $T_{2}$ contrast agent. To further evaluate their potential as an effective MRI probe for tumor, we attached a tumor-targeting ligand (folic acid) onto the micelle surface layer (Figure 2) and conducted the in vitro and in vivo MRI experiments. It is noteworthy that recently Qin et al reported a similar $T_{2}$ contrast agent prepared by coating SPION surface with Pluronic F127 copolymers. ${ }^{11}$ By comparison, SPIONs coated with the biodegradable PCL-PEG micelles should possess advantages in biosafety. In addition, the Pluroniccoated SPIONs do not have active targeting function for tumor, ie, no targeting ligand was introduced to the particle surface, and their in vivo performance was not tested yet in the mentioned literature.

\section{In vivo experiments}

So far, a wide variety of ligands such as antibodies and folic acid have been used to target cell-surface biomarkers in nanodelivery systems. This strategy enables nanoparticles to specifically enter malignant tumor cells, allowing an accurate diagnosis at a stage when the disease is still treatable. In the current study, we tested the potential of Fa-PEG-PCL-SPIONs for in vivo imaging applications. Magnetic micelle dispersions were injected into a nude mouse bearing the BEL-7402 tumor through the tail vein. Figure 6 shows the $T_{2}$-weighted images acquired before and after intravenous administration of Fa-PEG-PCL-SPIONs into a mouse, which demonstrated a great signal intensity decrease at a time point 3 hours after Fa-PEG-PCL-SPION administration. The effect of negative enhancement on the MRI $T_{2}$-weighted signal intensity of tumor upon injecting magnetic micelle solution into mice is summarized in Table 2. Regions of interest covering the entire tumor showed an average MRI signal intensity decrease
( $T_{2}$-weighted) of $41.2 \%$ at 3 hours after injecting Fa-PEGPCL-SPIONs, while only $16.4 \%$ decrease at this time point was observed when the same amount of nontargeting PEGPCL-SPIONs were applied $(n=6)$. Moreover, at 6 hours after injection, the $T_{2}$ signal drop for Fa-PEG-PCL-SPIONs was still as high as $32.4 \%$, suggesting an ideal postinjection time window for MRI scanning. By contrast, the $T_{2}$ signal drop for PEG-PCL-SPIONs was only $12.9 \%$ at this time point. The MRI $T_{2}$ signal intensity was slowly recovered, along with further extending postinjection time for MRI measurements. It is noteworthy that a similar type of time-dependent intratumor accumulation of an antibiofouling polymer-coated SPION has been reported. ${ }^{26}$ As shown in Figure 7, tumor tissues from Fa-PEG-PCL-SPION-administered mice showed a high content of ferric ion accumulation (B), while this was not the case in those tumor tissues from PEG-PCL-SPION-treated mice (A). The results indicate that the targeting of Fa-PEGPCL-SPIONs is very specific and in good agreement with the data obtained from MRI scanning.

In conclusion, our study demonstrate that Fa-PEG-PCLSPIONs not only have small particle size, but also high MRI sensitivity. Combining these characteristics with their specific tumor-targeting property, Fa-PEG-PCL-SPIONs can be used as a potential MRI contrast agent for in vivo tumor imaging.

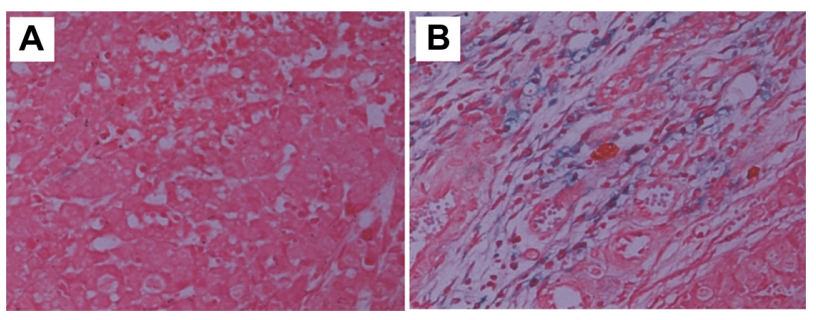

Figure 7 Prussian blue staining images of tumor tissues taken from mice at a time point 3 hours after injection of PEG-PCL-SPIONs (A) and Fa-PEG-PCL-SPIONs (B). Note: Blue stain density reflects the level of SPIO accumulation within tumor. Abbreviation: Fa-PEG-PCL-SPIONs, folate-attached poly(ethylene glycol)-poly( $\varepsilon$ caprolactone) superparamagnetic iron oxide nanoparticles. 


\section{Summary}

In summary, water-soluble and tumor-targeting SPIONs were synthesized by loading iron oxide ultrasmall nanoparticles into micelles of folate-PEG-PCL. The ultrasmall SPIOloaded micelles, Fa-PEG-PCL-SPIONs, are small (about $40 \mathrm{~nm}$ ) and favorable for long circulation and enhanced MRI $T_{2}$. Folate functionalization increases the cell uptake of SPION-loaded micelle. Furthermore, the in vivo MRI experiment and ex vivo histological study indicated that the Fa-PEG-PCL-SPIONs may build up in the tumor tissue, suggesting their potential in MRI diagnosis as a probe for folate receptor overexpressing tumor.

\section{Acknowledgments}

This research were supported by the National Natural Science Foundation of China (30900357 to Guo-bin Hong), the Scientific and Technologic Projects of Guangdong Province, China (2007B031516012 to Jing-xing Zhou), the Project Supported by Guangdong Natural Science Foundation, China (9451008901001949 to Guo-bin Hong), the Fundamental Research Funds for the Central Universities (10ykpy08 to Guo-bin Hong) and the Foundation for Distinguished Young Talents in Higher Education of Guangdong, China (LYM09006 to Guo-bin Hong).

\section{Disclosure}

The authors disclose no conflicts of interest.

\section{References}

1. Bautista MC, Miguel OB, Zhao X, et al. Comparative study of ferrofluids based on dextran-coated iron oxide and metal nanoparticles for contrast agents in magnetic resonance imaging. Nanotechnology. 2004;15:154-159.

2. Yu S, Chow GM. Carboxyl group $\left(-\mathrm{CO}_{2} \mathrm{H}\right)$ functionalized ferrimagnetic iron oxide nanoparticles for potential bio-applications. J Mater Chem. 2004; 14:2781-2786.

3. Sun $\mathrm{SH}$, Zeng H, Robinson DB, et al. Monodisperse $\mathrm{MFe}_{2} \mathrm{O}_{4}(\mathrm{M}=\mathrm{Fe}$, Co, Mn) nanoparticles. J Am Chem Soc. 2004;126:273-279.

4. Park J, An KJ, Hwang YS, et al. Ultra-large-scale syntheses of monodisperse nanocrystals. Nat Mater. 2004;3:801-805.

5. Wang Y, Wong JF, Teng XW, et al."Pulling" nanoparticles into water: phase transfer of oleic acid stabilized monodisperse nanoparticles into aqueous solutions of $\alpha$-cyclodextrin. Nano Lett. 2003;3:1555-1559.

6. Kim M, Chen YF, Liu YC, et al. Super-stable, high-quality $\mathrm{Fe}_{3} \mathrm{O}_{4}$ dendronnanocrystals dispersible in both organic and aqueous solutions. $A d v$ Mater. 2005;17:1429-1432.
7. Thünemann AF, Schütt D, Kaufner L, et al. Maghemite nanoparticles protectively coated with poly(ethylene imine) and poly(ethylene oxide)block-poly(glutamic acid). Langmuir. 2006;22:2351-2357.

8. Nikolic MS, Krack M, Aleksandrovic V, et al. Tailor-made ligands for biocompatible nanoparticles. Angew Chem Int Ed. 2006;45:6577-6580.

9. Tromsdorf UI, Bigall NC, Kaul MG, et al. Size and surface effects on the MRI relaxivity of manganese ferrite nanoparticle contrast agents. Nano Lett. 2007;7:2422-2427.

10. Yuan JJ, Armes SP, Takabayashi Y, et al. Synthesis of biocompatible poly[2-(methacryloyloxy)ethyl phosphorylcholine]-coated magnetite nanoparticles. Langmuir. 2006;22:10989-10993.

11. Qin J, Laurent S, Jo YS, et al. A high-performance magnetic resonance imaging $\mathrm{T}_{2}$ contrast agent. Adv Mater. 2007;19:1874-1878.

12. Ai H, Flask C, Weinberg B, et al. Magnetite-loaded polymeric micelles as novel magnetic resonance probe. Adv Mater. 2005;17:1949-1952.

13. Lee JH, Huh YM, Jun YW, et al. Artificially engineered magnetic nanoparticles for ultra-sensitive molecular imaging. Nat Med. 2007;13:95-99.

14. Huh YM, Jun YW, Song HT, et al. In vivo magnetic resonance detection of cancer by using multifunctional magnetic nanocrystals. $\mathrm{J}$ Am Chem Soc. 2005;127:12387-12391.

15. Leamon CP, Low PS. Folate-mediated targeting: from diagnostics to drug and gene delivery. Drug Discov Today. 2001;6:44-51.

16. Yang XQ, Deng WJ, Shuai XT, et al. Folate-functionalized polymeric micelles for tumor targeted delivery of a potent multidrug-resistance modulator FG020326. J Biomed Mater Res A. 2008;86:48-60.

17. Sahoo Y, Goodarzi A, Swihart MT, et al. Aqueous ferrofluid of magnetite nanoparticles: fluorescence labeling and magnetophoretic control. J Phys Chem B. 2005;109:3879-3885.

18. Jain TK, Morales MA, Sahoo SK, et al. Iron oxide nanoparticles for sustained delivery of anticancer agents. Mol Pharm. 2005;2:194-205.

19. Kim DK, Mikhaylova M, Wang FH, et al. Starch-coated superparamagnetic nanoparticles as MR contrast agents. Chem Mater. 2003; 15:4343-4351.

20. Weissleder R, Bogdanov A, Neuweltb EA, et al. Long-circulating iron oxides for MR imaging. Adv Drug Deliv Rev. 1995;16:321-334.

21. Chattopadhyay P, Gupta RB. Supercritical $\mathrm{CO}_{2}$ based production of magnetically responsive micro- and nanoparticles for drug targeting. Ind Eng Chem Res. 2002;41:6049-6058.

22. Ding DY, Hu Y, Zhang L, et al. Synthesis and magnetic properties of biocompatible hybrid hollow spheres. Biomacromolecules. 2006; 7:1766-1772.

23. Wang YJ, Hussain SM, Krestin GP. Superparamagnetic iron oxide contrast agents: physicochemical characteristics and applications in MR imaging. Eur J Radiol. 2001;11:2319-2331.

24. Nasongkla N, Bey E, Ren JM, et al. Multifunctional polymeric micelles as cancer-targeted, MRI-ultrasensitive drug delivery systems. Nano Letters. 2006;6:2427-2430.

25. Barbieri R, Quaglia M, Delfini M, et al. Investigation of water dynamic behaviour in poly(HEMA) and poly(HEMA-co-DHPMA) hydrogels by proton $\mathrm{T}_{2}$ relaxation time and self-diffusion coefficient n.m.r. measurements. Polymer. 1998;39:1059-1066.

26. Lee H, Lee E, Kim DK, et al. Antibiofouling polymer-coated superparamagnetic iron oxide nanoparticles as potential magnetic resonance contrast agents for in vivo cancer imaging. $J$ Am Chem Soc. 2006;128:7383-7389.
International Journal of Nanomedicine

\section{Publish your work in this journal}

The International Journal of Nanomedicine is an international, peerreviewed journal focusing on the application of nanotechnology in diagnostics, therapeutics, and drug delivery systems throughout the biomedical field. This journal is indexed on PubMed Central, MedLine, CAS, SciSearch ${ }^{\circledR}$, Current Contents ${ }^{\circledR} /$ Clinical Medicine,

\section{Dovepress}

Journal Citation Reports/Science Edition, EMBase, Scopus and the Elsevier Bibliographic databases. The manuscript management system is completely online and includes a very quick and fair peer-review system, which is all easy to use. Visit http://www.dovepress.com/ testimonials.php to read real quotes from published authors. 